\title{
Anatomical and surgical evaluation of gastroepiploic artery
}

\author{
By
Kodai TOMIOKA ${ }^{1,2 *}$, Masahiko MURAKAMI', Akira SAITO ${ }^{1,2}$, Hiromitsu EZURE ${ }^{1}$, Hiroshi MORIYAMA ${ }^{1}$, Ryoichi MORI ${ }^{1}$ and Naruhito OTSUKA ${ }^{1}$
${ }^{1}$ Department of Anatomy, Showa University School of Medicine, 1-5-8 Hatanodai, Shinagawa, Tokyo 142-8555, Japan
${ }^{2}$ Department of Gastroenterological and General Surgery, Showa University Hospital, 1-5-8 Hatanodai, Shinagawa, Tokyo 142-8666, Japan

-Received for Publication, December 21, 2015-

\begin{abstract}
Key Words: gastroepiploic, artery, anatomy, surgery
Summary: Objective: Knowledge of the anatomy of the stomach and its surrounding structures is essential for lymph node dissection. This is the first gross anatomical investigation of anastomosis variations in the gastroepiploic arteries (GEA). The aim of this study is to examine the anastomosis pattern of the right and left GEA (RGEA and LGEA, respectively). Methods: Seventeen cadavers were dissected, and the anastomotic patterns of RGEA and LGEA were observed macroscopically. Results: The anastomotic patterns were classified into two groups. Type 1 had direct anastomosis $(n=16 ; 94.1 \%)$, whereas Type 2 had no anastomosis $(n=1 ; 5.9 \%)$. Formation of an arterial arch along the greater curvature was observed in twelve cases $(\mathbf{7 0 . 6 \%})$, whereas four cases $(\mathbf{2 3 . 5 \% )}$ ) exhibited mesh-like anastomosis or narrow anastomotic branches. Conclusions: Direct anastomoses were observed in almost all the cases. These results are significant, and may be useful in the classification of lymph nodes or speculation of cancer metastases.
\end{abstract}

\section{Introduction}

Gastrectomy with lymph node dissection is an essential procedure performed by gastroenterological surgeons for the treatment of gastric cancer. In this field, the lymph nodes are distributed along the arteries and defined according to the Japanese Classification of Gastric Carcinoma, 14th Edition [1]. The greater curvature, in particular, shows the lymph nodes on the borders of the anastomosis of the left and right gastroepiploic arteries (GEA). Lymph nodes in the area of the left GEA (LGEA) are defined as $4 \mathrm{sb}$, whereas those in the area of the right GEA (RGEA) are defined as 4d [1]. Therefore, surgeons rely on this arterial boundary to distinguish lymph nodes. However, different anastomotic forms of the GEA arterial anastomosis have been described in anatomical texts and surgical manuals. Some reports clear anastomosis with the formation of a great arterial arch, whereas others state that they are completely independent on each other. Furthermore, there are no gross anatomical studies investigating variations in the anastomotic form of the gastroepiploic artery. For surgeons, knowledge on the existence of this arterial anastomosis, and the ability to identify it macroscopically is of utmost importance. In this study, we examined the gastroepiploic arterial anastomotic form using gross anatomical methods on adult cadavers. Furthermore, this study must be useful to make a basic data to depict correctly the GEA form in the anatomical and surgical textbooks.

\section{Materials and Methods}

Twenty-eight adult cadavers were dissected at the Showa University in 2014. Seventeen cadavers [five males and twelve females, median age: 82 years, (68-95 years)], with no surgical history involving the stomach, were selected for this study. All cadavers were fixed with $10 \%$ formalin solution. The stomach, duodenum, spleen, celiac artery, common hepatic artery, splenic artery, gastroduodenal artery, and right and left gastroepiploic arteries were excised en-bloc. After excision, the adipose and connective tissues around the stomach and arteries were removed, and the RGEA and LGEA were examined 
Table 1. Anastomosis classification of right and left gastroepiploic arteries.

\begin{tabular}{cccc}
\hline Type & & Number & Percentages (\%) \\
\hline Type 1 & & 16 & 94.1 \\
& Arterial arch & 12 & 70.6 \\
& Fine anastomosis & 4 & 23.5 \\
Type 2 & & 1 & 5.9 \\
\hline
\end{tabular}

carefully. The thin arteries that were visible macroscopically were retained. After dissection, we observed the anastomotic forms of RGEA and LGEA, and classified them into two groups.

\section{Result}

In all the cadavers, RGEA was seen to branch from the gastroduodenal artery, whereas LGEA branched from the splenic artery. Obvious mutation was not recognized.

\section{Anastomosis pattern of the arteries}

Anastomosis patterns were classified into the following two types:

Type 1: The direct anastomotic type.

Type 2: The completely independent type.

In our study, there were sixteen cases (94.1\%) of Type 1 , and only one case (5.9\%) exhibiting Type 2 . Within the Type 1 category, twelve cases $(70.6 \%)$ were seen to form an arterial arch along the greater curvature, whereas the remaining four cases $(23.5 \%)$ exhibited mesh-like anastomosis or narrow anastomotic branches (Table 1) (Figure $1)$.

\section{Discussion}

The stomach has its developmental origin in the foregut, and it is supplied by branches of the celiac artery. Its distribution is divided into the greater curvature side, supplied by the right gastric artery, and the lesser curvature side, supplied by RGEA, LGEA, and short gastric artery. RGEA and LGEA run along the greater curvature of the stomach, at a distance of about $1 \mathrm{~cm}$ from it, and form an arterial arch anastomosing at the middle of the stomach [2]. Although Adachi described the branching pattern of the celiac arterial distribution [3], there are no gross anatomical studies about GEA, which is the peripheral artery of the celiac artery. All currently available information on this artery is from biological or surgical specimen evaluations using angiography and 3-dimensional computed tomography [4-8]. Furthermore, there is no unified view on the anastomosis patterns of GEA. Some anatomical texts and surgical manuals report that RGEA and LGEA form an arterial arch with the same thickness [9-13], whereas others report that the two are

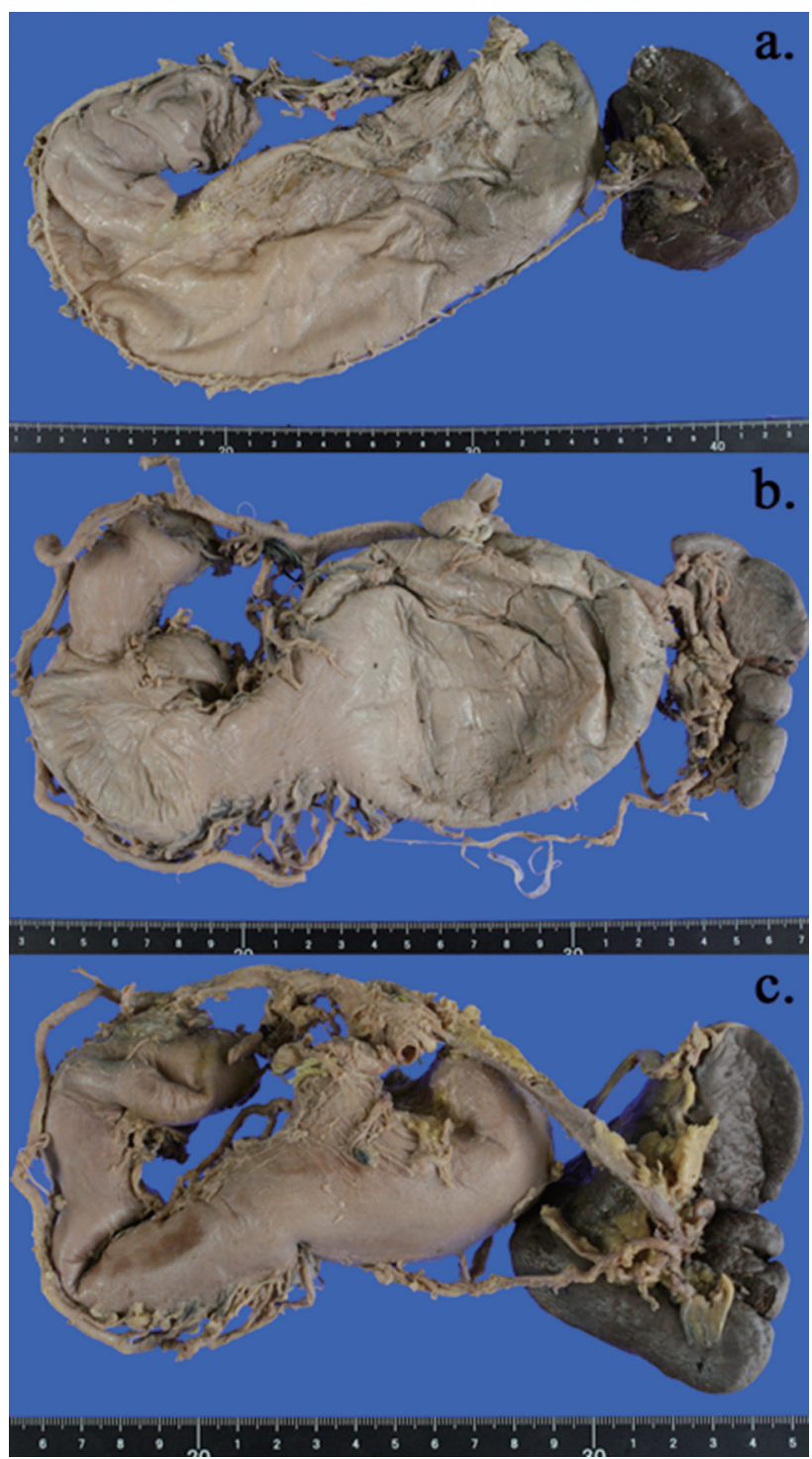

Fig. 1. Anastomosis pattern of gastroepiploic arteries. a: Direct anastomosis with arterial arch. b: Direct anastomosis with fine branch. c: Arteries were independent of each other.

independent of each other [14, 15]. However, according to the Japanese Classification of Gastric Carcinoma, 14th Edition, the lymph nodes belonging to RGEA and LGEA areas are defined as number $4 \mathrm{~d}$ and $4 \mathrm{sb}$, respectively [1]. Thus, it is necessary to fix the arterial boundary as a landmark to enable identification of the lymph nodes. This is the first study to examine anastomotic patterns of RGEA and LGEA, using the gross anatomical approach. Our results showed that direct anastomoses of RGEA and LGEA existed in almost all of the cases. Of this, $70 \%$ of the cases exhibited formation of an arterial arch along the greater curvature. In addition, there were very few cases where RGEA and LGEA were independent of each other and did not exhibit anastomosis. A literature search 
Table 2. Literature review of anastomosis variations in gastroepiploic arteries.

\begin{tabular}{|c|c|c|c|c|c|c|c|c|}
\hline Author & Year & Country & Language & Number & Method & $\begin{array}{l}\text { Clear continuous } \\
\qquad(\%)\end{array}$ & $\begin{array}{l}\text { Anastomosis } \\
\text { branch }(\%)\end{array}$ & $\begin{array}{c}\text { Not anastomosis } \\
(\%)\end{array}$ \\
\hline Levasseur et al. [7] & 1968 & France & French & 47 & Celiac angiogram & 37.2 & 27.8 & 35.0 \\
\hline Yamato et al. [8] & 1979 & Japan & English & 137 & Celiac angiogram & 34.4 & 15.3 & 50.3 \\
\hline Hannoun et al. [9] & 1984 & France & English & 27 & Angiogram & 23.5 & 20.0 & 46.5 \\
\hline Our cases & 2015 & Japan & Engilsh & 17 & Dissection & 70.6 & 23.5 & 5.9 \\
\hline
\end{tabular}

in PubMed revealed only three reports that used angiograms [Table 2]. According to Levasseur et al., 37.2\% cases showed clear continuous anastomosis, 27.8\% showed anastomosis by thin artery, and $35.0 \%$ exhibited no anastomosis and absence of aortic arch anastomosis in the greater curvature [5]. Yamato et al. reported that $34.5 \%$ cases showed direct extramural communication, $15.0 \%$ exhibited fine reticular anastomoses, $44.5 \%$ lacked anastomoses altogether, and $6.0 \%$ presented with indirect anastomoses along the omental branches [6]. These results were in agreement with those of Hannoun et al. [7]. According to these reports, clear anastomosis and arterial arch formation account for $23.5 \%-37.2 \%$ of cases, which was largely different from the results of this study. Moreover, we observed no anastomosis in 5.3\% (n $=1$ ) of cases, whereas other studies reported higher rates of $35.0 \%-50.5 \%$. These differences were attributed to the use of gross anatomical methods and X-ray fluoroscopy with a contrast medium. The main concern with the former method was vascular damage during dissection and misidentification. However, vascular damage was considered to be minimized in this study, as the roots of RGEA and LGEA, branching from the gastroduodenal and splenic artery, were identified and dissected carefully along the greater curvature by the same author group. Some of the concerns with the latter method include existence of arteries through which the contrast medium cannot pass and thin arteries or anastomotic branches with arterial lumen diameter $<1 \mathrm{~mm}$, making it difficult to recognize them on an angiogram. Moreover, the presence or absence of contrast in peripheral fine angiography varies with injection pressure at the time of inspection, hemodynamics, and the patient's condition. Actually, we previously reported the length, circumference, area and major axis of GEA for the three points; RGEA, LGEA and the anastomotic point [16]. The mean major axis of the arterial lumen at the anastomotic point was $1.00 \pm$ $0.56 \mathrm{~mm}$. One study reported that insufficient contrast could not be labeled as blockage or constriction immediately, as an absence of contrast did not necessarily reflect the blood flow [17]. This fact was considered similar possibilities for peripheral angiography of the abdominal area, and that could be a basis for that an absence of contrast imaging could not prove that the vessel did not exist. Moreover, the major axis of the thinnest artery at the anastomosis point was $0.3 \mathrm{~mm}$, and could make it difficult to recognize it under X-ray fluoroscopy. Based on these results, this study, using gross anatomical approach, was considered to be more meaningful.

It was difficult to determine the boundaries of the lymph nodes $4 \mathrm{sb}$ and $4 \mathrm{~d}$ in the cases forming an arterial arch $(70 \%)$, as the lymph nodes are defined by the artery course in gastric cancer. The lymph nodes No. $4 \mathrm{sb}$ and $4 \mathrm{~d}$ were in a D1 dissection group regardless of the surgical procedure for gastric cancer, and their impacts on the prognosis provision were equivalent [1]. Therefore, we propose that GEA cases with clear boundaries follow the conventional lymph node classification, whereas those with unclear boundaries are classified into three major groups (RGEA roots, LGEA roots, and greater curvature). In cardiovascular fields, RGEA is widely used as an arterial graft for coronary artery bypass grafting [18], and we hope our study will contribute positively.

Our study has certain limitations. We performed dissection in only 17 cadavers, which may have led to a selection bias. A larger sample size would help overcome these limitations.

\section{Conclusion}

We examined GEA anastomosis patterns using gross anatomical methods, for the first time. Our results showed that direct anastomosis of RGEA and LGEA was observed in almost all cases, and formation of arterial arch in about $70 \%$ of the cases. These results are significant, and may be useful in the classification of lymph nodes or speculation of cancer metastases. The frequency of anastomosis reported by us significantly differed from previous published papers, indicating the importance of gross anatomical studies. However, more studies using larger sample sizes are required.

\section{Acknowledgement}

We thank the patients for allowing us to publish this study, and providing informed consent for this study.

\section{References}

1) Japanese gastric cancer association. Japanese classification of gastric carcinoma (The 14th edition). 2010. 
2) Testut L, Latarjet A. Traite d'anatomie humaine. Doin. Paris.1949.

3) Adachi B. Das Arteriensystem der japaner. Vol. 2. Kyoto. 1928; 42-46.

4) El-Eishi HI, Ayoub SF, Ade-el-Khalek M. The arterial supply of the human stomach. Acta. Anat. 1973; 86 (3): 565-580.

5) Levasseur JC, Couinaud C. Etude de la distribution des arteres gastriques. J Chir (Paris). 1968; 95 (1): 57-78.

6) Yamato $T$, Hamanaka Y, Hirata S, Sakai K. Osophagoplasty with an autogenous tubed gastric flap. Am. J. Surg. 1979 May; 137 (5): 590-602.

7) Hannoun L, Breton CL, Bors V, Helenon C, Bigot JM, Parc R. Radiological anatomy of the right gastroepiploic artery. Anat. Clin. 1984; 5 (4): 265-271.

8) Kobayashi T, Ikeda Y, Murakami M, Shirakawa B, Ito H, Mikamo A, et al. Computed tomographic angiography to evaluate the right gastroepiploic artery for coronary artery bypass grafting. Ann. Thorac. Cardiovasc. Surg. 2008; 14 (3): 166-171.

9) Clemente CD. Gray's anatomy thirties American edition. LEA \& Febiger Philadelphia. 1985; 733-735.

10) Thorek, P. Anatomy in surgery third edition. Springer-verlag New York. 1985; 434-455.

11) Morris PJ, Malt RA. Oxford textbook of surgery volume 1. Oxford medical publications. 1994; 931-942.

12) Netter F. Atlas of human anatomy fourth edition. Philadelphia: Saunders Elsevier. 2011; P305.

13) Zollinger RM Jr., Ellison EC. Zollinger's atlas of surgical operations international edition. The McGraw hill medical. 2011; $67-71$.

14) Soper NJ, Swanstrom LL, Eubanks WS. Mastery of endoscopic and laparoscopic surgery third edition. Wolters Kluwers Lippincott Williams and Wilkins. 2009; 239-248.

15) Nyhus LM, Baker RJ. Mastery of surgery second edition Vol 1. Little, Brown and company. 1992; 625-632.

16) Tomioka K, Murakami M, Saito A, Ezure H, Moriyama H, Mori R, Otsuka N. Morphometric and quantitative evaluation of the gastroepiploic artery. Okajimas Folia Anat Jpn. 2015; 92 (2): 33-35.

17) Mochizuki $H$, Yamanouchi $H$, Tohgi $H$, Tomonaga $M$. The variation of the basal arteries of the brain: Their frequency, laterality, and clinical significance in cerebral angiography (article in Japanese). Jpn. J. Stroke. 1980; 2 (4): 382-387.

18) Pym J, Brown PM, Carrette EJ, Parker JO, West RO. Gastroepiploic-coronary anastomosis. A visible alternative bypass graft. J. Thorac. Cardiovasc. Surg. 1987; 94 (2): 256-259. 